\title{
Challenges Toward Measles Elimination
}

\author{
Masoud Mardani (ii) ${ }^{1, *}$ \\ ${ }^{1}$ Infectious Diseases and Tropical Medicine Research Center, Shahid Beheshti University of Medical Sciences, Tehran, Iran \\ "Corresponding author: Infectious Diseases and Tropical Medicine Research Center, Shahid Beheshti University of Medical Sciences, Tehran, Iran. Email: \\ drmasoudmardani@yahoo.com \\ Received 2019 May 25; Accepted 2019 June 01.
}

Keywords: Measles, Elimination, Vaccination

Recently updated data show that at least 159 cases of measles have been confirmed in the United States of America in 2019, that is in fact an unwelcome resurgence of viral infection, which had been eliminated in 2000. Alexander said "Charlants and internet fraudsters who claim that vaccines aren't safe are preying on unfounded fears and daily struggles of parents, and they are creating a public health hazard that is entirely preventable" (1). Measles is a highly contagious infectious disease caused by the measles virus, it is worrisome and could spread through coughing and sneezing, for two hours it can linger in the surface and air (2). In spite of the protected and viable measles antibody being accessible and regardless of endeavors put in by different partners in the course of recent decades, measles keeps on killing 360 adolescents consistently around the world. Controlling and taking out measles, which was executing more than 6800 kids yearly, was mooted during the 1980s, not long after the destruction of smallpox (3). In spite of the fact that these set targets were not accomplished because of holes in inoculation; the measles end and control programs spared a huge number of lives by counteracting hazardous inconveniences and continuations, (for example, bronchopneumonia, extreme intense ailing health, military tuberculosis, keratomalacia, and subacute sclerosing panencephalitis) in a few additional adolescents through improved vaccination inclusion. In 2010, the World Health Assembly set three achievements for measles counteractive action to be accomplished by 2015: (1) increment routine inclusion with the primary portion of measles-containing immunization (MCV1) among kids matured one year to $\geq 90 \%$ at the national dimension and to $\geq 80 \%$ in each region; (2) decrease worldwide yearly measles occurrence to under five cases for each million populace; (3) decrease worldwide measles mortality by $95 \%$ from the 2000 gauge. In 2012, the World Health Assembly embraced the Global Vaccine Action Plan (GVAP), with the target of taking out measles in four of the six World Health Organization (WHO) locales by 2015 and in five districts by 2020 (4). Although this action leads to prevention of 21.1 million deaths by measles vaccination in that period, however, measles disposal achievements have not been met and three districts are encountering an enormous measles resurgence. To gain further ground, casebased reconnaissance should be reinforced, and inclusion with MCV1 and the second portion of measles-containing antibody (MCV2) needs to increment; moreover, it will be essential to keep up political responsibility and guarantee generous, supported speculations to accomplish worldwide and local measles end objectives (4). In a report from the United Nations, an excess of 20 million adolescents a year passed up measles antibodies over the world in the previous eight years, laying a way of introduction to an infection that is presently causing illness flare-ups all inclusive. Measles is an exceedingly infectious ailment that can slaughter and can cause visual deficiency, deafness or cerebrum harm. It is as of now spreading in flare-ups in numerous pieces of the world, incorporating into the United States, Europe, the Philippines, Tunisia, and Thailand. Two dosages of the measles antibody are basic to secure adolescents and the WHO says 95\% immunization inclusion is required for "crowd invulnerability" against measles. In any case, due to the of absence of access, weakness frameworks, lack of concern, and sometimes dread or wariness regarding vaccines, the worldwide inclusion of the main portion of the measles antibody was accounted for at $85 \%$ in 2017 - a dimension that has stayed comparative for as far back as decade. Worldwide inclusion for the second portion is even lower, at $67 \%$. Among high income countries the United States, which had the measles outbreak in areas of stricter immunization of California, most children were missing the first dose of vaccine between 2010 and 2017, at more than 2.5 million. Next came France and Britain, with 
more than 600,000 and 500,000 unvaccinated children, respectively during the same period. However, the situation in $\mathrm{n}$ poorer countries is critical for example Nigeria in 2017 had the most elevated number of adolescents under one year old who passed up the primary portion, at almost 4 million. It was trailed by India with 2.9 million, Pakistan and Indonesia with 1.2 million each, and Ethiopia with 1.1 million (5).

In Iran, due to the Extended Program of Immunization (EPI) there is 99\% coverage of measles vaccination and the country is moving towards eradication of measles. However, what has happened in all parts of the world shows that there is risk of measles resurgence even after elimination, therefore there is a need for precise planning and community-based training to prevent the onset of illness. According to UNICEF, measles was far too contagious a disease to be ignored and urged health officials to do more to fight it.

\section{Footnotes}

Conflict of Interests: It is not declared by the author.
Funding/Support: It is not declared by the author.

\section{References}

1. Troy Brown RN. US measles cases now the highest since eliminated in 2000. Medscape Medical News; 2019. Available from: https://www. medscape.com/viewarticle/912215.

2. World Health Organization. Measles. Immunization, vaccines and biologicals. World Health Organization; 2018. Available from: https:// www.who.int/immunization/diseases/measles/en/.

3. Bavdekar SB, Karande S. Elimination of measles from India: Challenges ahead and the way forward. J Postgrad Med. 2017;63(2):75-8. doi: 10.4103/jpgm.JPGM_166_17. [PubMed: 28397737]. [PubMed Central: PMC5414430].

4. Dabbagh A, Laws RL, Steulet C, Dumolard L, Mulders MN, Kretsinger $\mathrm{K}$, et al. Progress toward regional measles elimination - worldwide, 2000-2017. MMWR Morb Mortal Wkly Rep. 2018;67(47):1323-9. doi 10.15585/mmwr.mm6747a6. [PubMed: 30496160]. [PubMed Central: PMC6276384].

5. Kelland K. Millions of children miss measles shots, creating outbreaks: UNICEF. Reuters; 2019. Available from: https://www.reuters.com/ article/us-health-measles-global/millions-of-children-missmeasles-shots-creating-outbreaks-unicef-idUSKCN1S1004. 